\title{
Familial Otosclerosis Associated with Osteogenesis Imperfecta: A Case Report
}

\author{
Ha Neul Lee, Hyun Jong Jeon, and Young Joon Seo \\ Department of Otorhinolaryngology-Head and Neck Surgery, Research Institute of Hearing Enhancement, Yonsei University \\ Wonju College of Medicine, Wonju, Korea
}

\author{
Received February 23, 2021 \\ Revised March 25, 2021 \\ Accepted April 8, 2021 \\ Address for correspondence \\ Young Joon Seo, MD, PhD \\ Department of Otorhinolaryngology- \\ Head and Neck Surgery, \\ Yonsei University \\ Wonju College of Medicine, \\ 20 Ilsan-ro, Wonju 26426, Korea \\ Tel $+82-33-741-0642$ \\ Fax +82-33-732-8287 \\ E-mail okas2000@hanmail.net
}

Otosclerosis, a hereditary disorder characterized by disordered resorption and deposition of bone, results in progressive hearing loss. Osteogenesis imperfecta $(\mathrm{Ol})$ is a genetic disorder characterized by recurrent fractures, blue sclera, and varying degrees of hearing impairment; and is a known risk factor for otosclerosis. After adolescence, the risk of fracture decreases, reducing the need for follow-up in Ol. However, otosclerosis is a progressive disorder. In this report, we discuss two cases of familial otosclerosis with different clinical features. We hypothesize that the difference in hearing level correlates with the difference in computed tomography findings. The mother, whose case was considered severe, was prescribed hearing aids, while the daughter, who had normal hearing level, was regularly followed up.

J Audiol Otol 2021;25(4):230-234

Keywords: Otosclerosis; Familial otosclerosis; Osteogenesis imperfecta; Hearing loss; Hereditary disorder.

\section{Introduction}

Otosclerosis, also known as otospongiosis, is a disease characterized by progressive hearing loss caused by pathologic bone remodeling of the bony labyrinth, especially the otic capsule [1,2]. The disease mainly involves decreased mobility of the stapedial foot plate leading to conductive hearing loss. As the disease progresses, the bony labyrinth undergoes remodeling and causes a mixed type of hearing loss on audiogram. Patients usually present with hearing impairment, but the presenting symptom could be dizziness, tinnitus or other otologic symptoms.

Otosclerosis is found in $12 \%$ of the Caucasian population but is less common in African and Asian populations. It is also found to be 1.5 times to 2 times more prevalent in females $[2,3]$. In Korea, otosclerosis is a clinically rare disease; thus, many patients may either be underdiagnosed or misdiagnosed. There are also few studies about otosclerosis in Korean; only one previous case about siblings with otosclerosis in Korea

This is an Open Access article distributed under the terms of the Creative Commons Attribution Non-Commercial License (https:/creativecommons.org/licenses/by-nc/4.0/) which permits unrestricted non-commercial use, distribution, and reproduction in any medium, provided the original work is properly cited. has been reported [4].

Osteogenesis imperfecta (OI) is a heritable syndrome characterized by one or more of the features of osteoporosis leading to fractures, blue sclera, hyperlaxibility, and impaired hearing present throughout life. Hearing impairment is a common finding in the patients diagnosed with OI [5]. In this study, we report two cases of otosclerosis with OI and their management.

\section{Case Report}

\section{Patient 1}

A 41-year-old female visited our outpatient center for progressive hearing loss in both ears since the previous year. She was aware that her hearing ability was suboptimal since childhood. She only complained about hearing difficulty; no tinnitus, otalgia, or dizziness were noted. Her past medical history revealed that she had repeated fractures in her long bones when she was child. There was no other medico-surgical history. On physical examination, there was an increase in vascularity of the promontory of both tympanic membranes (positive Schwartze sign) (Fig. 1A); both external auditory canals appeared normal. 
On pure tone audiometry (PTA), average thresholds of air conduction for $0.5,1,2$, and $4 \mathrm{kHz}$ were 64 and $52 \mathrm{~dB}$ for the right and left ears, respectively. Average threshold of bone conduction was $25 \mathrm{~dB}$ for the right ear. There was no airbone gap noted for the other ear (Fig. 2A). There was a peak of bone conduction threshold at $2,000 \mathrm{~Hz}$, which seem to be Carhart's notch associated with stapedial fixation on the right ear. Phonetically balanced (PB) max scores (maximum score on a performance-intensity functions for PB word lists) in speech audiometry were $60 \%$ and $96 \%$ in the right and left ears, respectively. Both tympanic membranes showed type A tympanometry results.

There was an opaque lesion found around both bony labyrinths on temporal bone computed tomography (CT), suggestive of otosclerosis (Fig. 3A-C). We prescribed receiver-in-canal type hearing aids for both ears. There was an objective hearing gain noted on the audiogram and in the language assessment. There was also a note of subjective hearing improvement in her daily life.

\section{Patient 2}

A 14-year-old girl, daughter of patient 1, visited our outpatient center for hearing screening. Her past medical history revealed repeated fractures in her lower limbs. She was diagnosed with OI at another hospital. She had no otologic symptoms; both tympanic membranes and external auditory canals appeared normal on physical examination (Fig. 1B).

PTA revealed average thresholds of air conduction of $10 \mathrm{~dB}$ for the right ear and $15 \mathrm{~dB}$ for the left ear (Fig. 2B). On temporal bone $\mathrm{CT}$, spongiotic changes were noted on both cochleas (Fig. 3D-F). We regularly followed her up without treatment because she had no subjective symptoms and hearing ability was within normal range. No other people from her family were noted to suffer from hearing difficulty (Fig. 4).
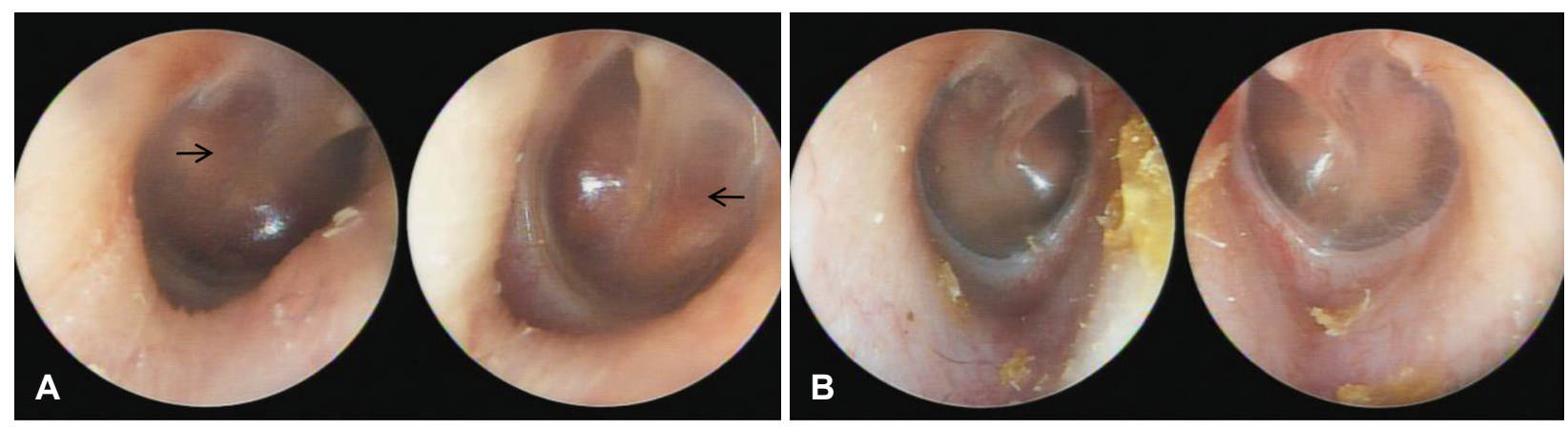

Fig. 1. Tympanoscopic image of both cases. A: Tympanoscopic image of patient 1 . Arrows indicate hypervascularization of the promontory of both tympanic membranes (positive Schwartze sign). B: Tympanoscopic image of patient 2. There was no specific abnormality.
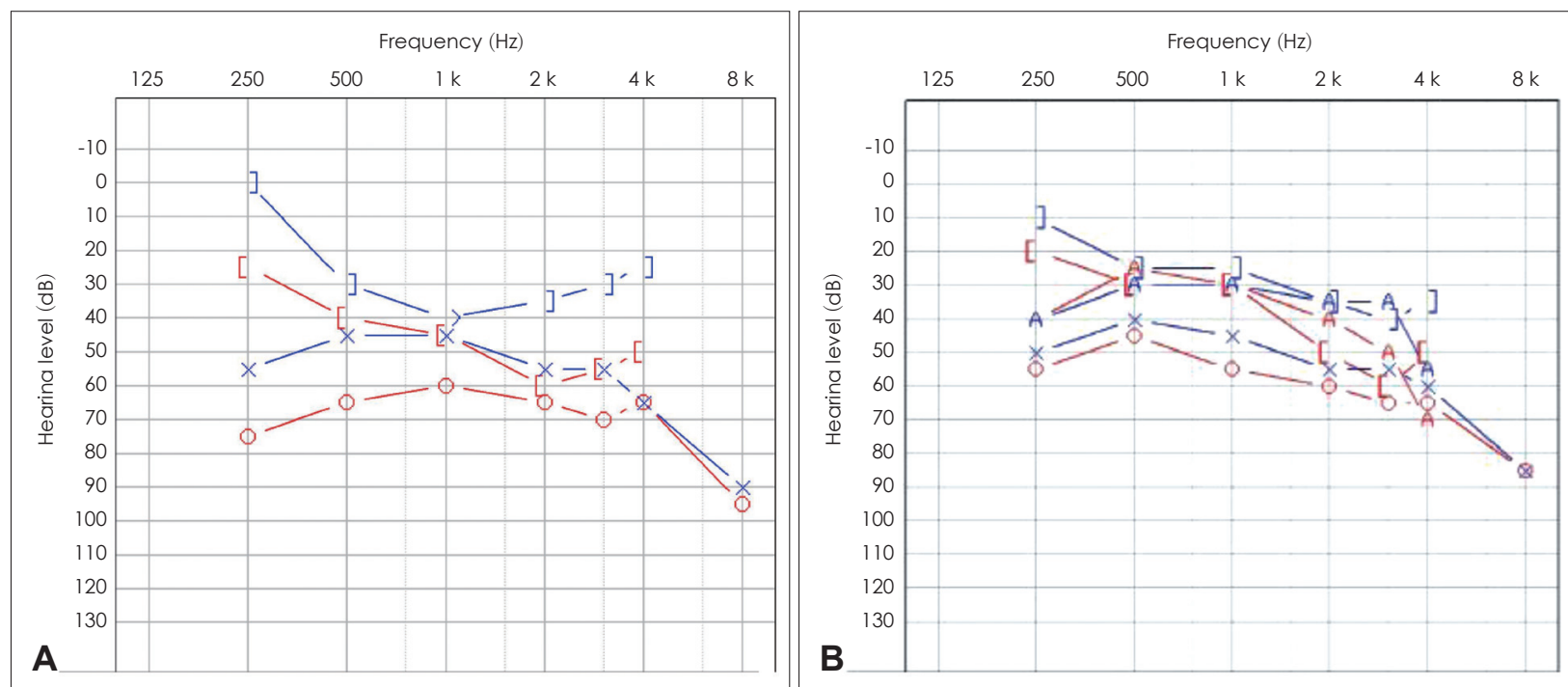

Fig. 2. PTA of both cases. A: Initial PTA of patient 1. The right ear shows $2,000 \mathrm{~Hz}$ bone conduction threshold dip known as Carhart's notch. It is a well known indicator of stapedial fixation. B: PTA of patient 2. PTA: pure tone audiogram. 

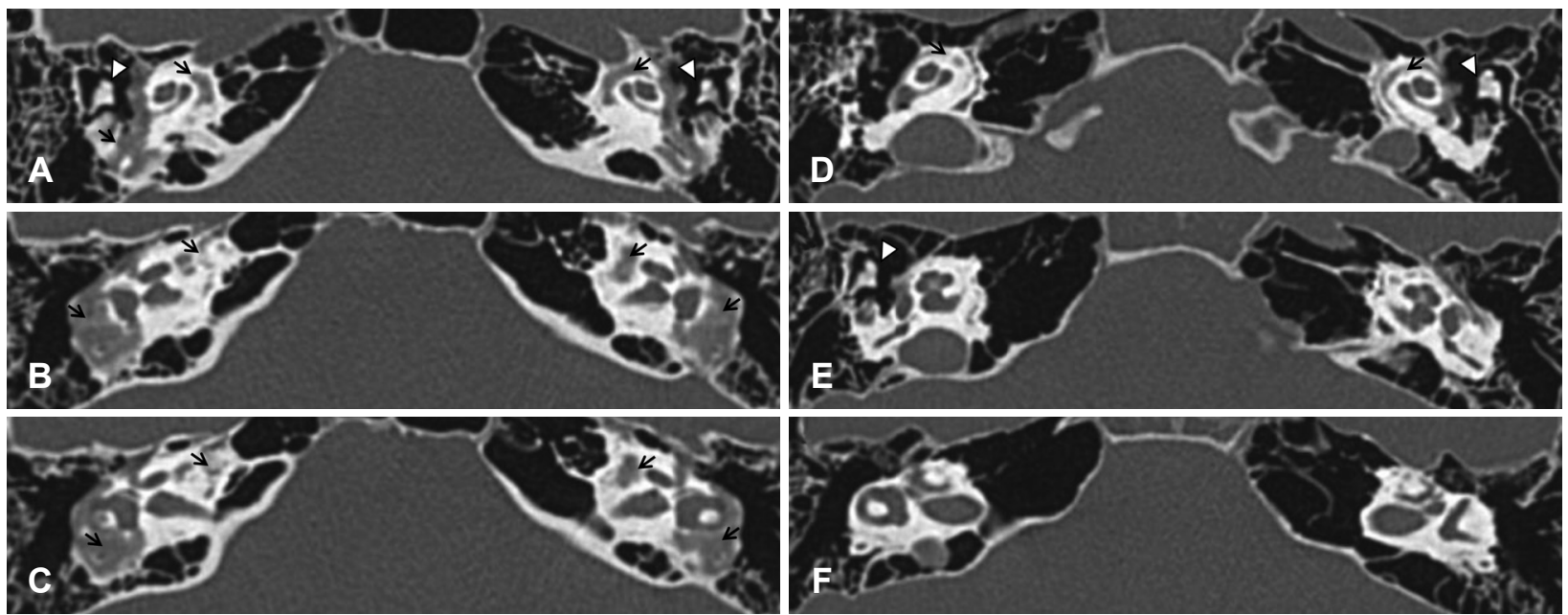

Fig. 3. Axial view of temporal bone CT. A, B, C: Serial view of temporal bone CT of patient 1. Arrows indicate more extensive demineralization of both bony labyrinths than that of patient 2. Arrowheads indicate the incus of ossicle chains. D, E, F: Serial view of temporal bone CT of patient 2 . There is an evidence of demineralization change of both bony labyrinths (arrows). Arrowheads indicate the incus of ossicle chains. CT: computed tomography.

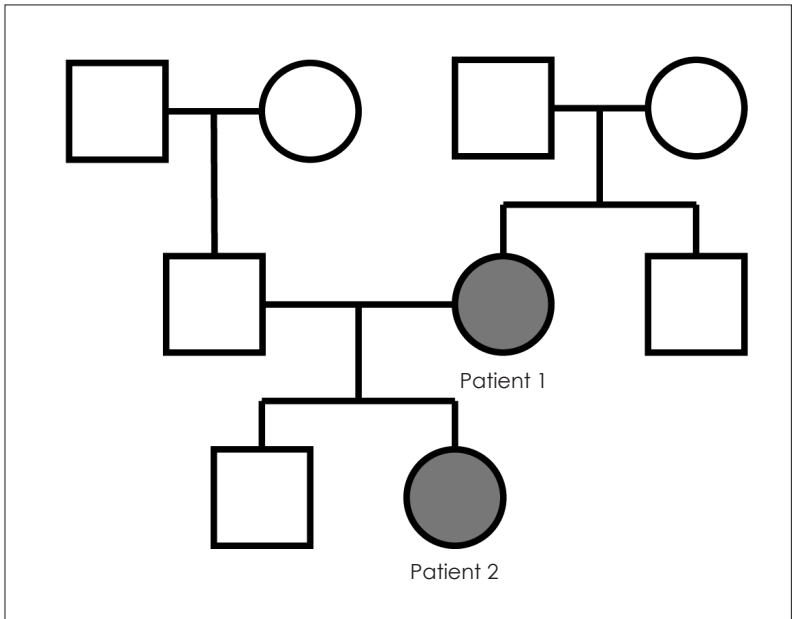

Fig. 4. Pedigree of our cases. There are no other people suffer from hearing difficulty except our cases.

\section{Discussion}

The diagnosis of otosclerosis can be made histopathologically after stapedeotomy or stapedectomy. However, the diagnosis is usually made clinically by history, physical examination, audiogram results, and temporal bone CT. Sixty percent of patients suffering from otosclerosis report familial history, as seen in our cases [1]. The disease has an autosomal-dominant inheritance pattern with an incomplete penetration. With the advancement of radiologic technique, high-resolution CT (HRCT) has been used for diagnosis and surgical management of otosclerosis [1]. Typical findings on HRCT include radiolucency within the otic capsule, thickening of the stapes, and demineralization of the area outlining the cochlea ("double-ring" sign), as seen in patient 1 . These features have high diagnostic sensitivity and specificity.

$\mathrm{OI}$ is a hereditary syndrome resulting from genetic mutation of collagen production and secretion. OI was originally classified by Sillence into four types according to inheritance, features, and severity [5]. The most frequent type of OI encountered by otolaryngologists is OI type I, which is due to mutations in the COL1A1 or COL1A2 gene. Patients with OI present with repeated fractures and hearing impairment, as seen in our cases; hence, OI is a known risk factor for otosclerosis. However, there is no association between the genotype and hearing ability. A previous review of the histopathological changes of temporal bones of patients diagnosed with OI through the National Temporal Bone Registry database of America described the temporal bone lesions and their implications on the observed heterogeneity of hearing loss patterns [6]. The otopathologic changes in otosclerosis cases vary from histologic otosclerosis to the fixation of the foot plate of stapes and even cochlear otosclerosis [6,7].

Surgery is an effective option for the management of otosclerosis. Stapedotomy or stapedectomy increases the mobility of the involved stapedial foot plate [8]. It should be considered in patients with conductive hearing loss of $20 \mathrm{~dB}$ or more, $\mathrm{PB}$ max of $60 \%$ or more, and with fair general condition. However, patients must be informed that otosclerosis is a progressive disease even with successful surgery. Around $10-20 \%$ of patients may need to undergo reoperation $[9,10]$. Meanwhile, hearing aid technology could also be a good treatment option for patients who are unbale to undergo surgery or who have sensorineural hearing loss of $25 \mathrm{~dB}$ or more [1].

Furthermore, due to the advancement in hearing rehabilitation surgery, middle ear implant (MEI), bone conduction im- 
plant, and cochlear implant (CI) could be successful alternative treatment options for otosclerosis [11]. MEI could be an effective surgical option in patients with intact ossicle mobility; hence, this surgery is performed with or after stapedial surgery [12]. Physicians may consider bone conduction implant in patients with mixed type hearing impairment or mild sensorineural hearing loss. However, in these cases, hearing aids must be tried first. CI could be a successful strategy for advanced cases. However, factors such as the structural change of the inner ear and the possibility of reoperation must be considered before surgery $[13,14]$.

Some physicians recommend oral administration of sodium fluoride due to its antagonistic effect on bone remodeling and osteoclastic activation. However, there is no clinical consensus on this [15]. Some studies have been conducted on bisphosphonate and vitamin $\mathrm{D}$ derivatives, but have not proven established treatment modalities for otosclerosis [15].

Treatment for OI type I mainly focuses on the prevention of fractures, physical therapy, and rehabilitation exercise. The risks of fracture decreases after adolescence, and, usually, there is no need for regular follow-up on skeletal concerns. However, patients with OI may have progressive impairment. For this reason, otolaryngologists should regularly follow-up patients to provide an appropriate treatment strategy at the earliest.

In both our cases, we could easily diagnose otosclerosis clinically without genotyping. Patient 2 had already been diagnosed with OI. Patient 1 had not yet been diagnosed with OI before. However, given her childhood history of repeated fractures, we presume that she also had OI. Therefore, both cases had OI as a risk factor for otosclerosis and both were informed of the need for regular follow-ups because of the progressive nature of the disease. In patient 1, the patient's audiogram showed moderate hearing impairment of both ears with conductive hearing loss of the left ear. Our treatment strategy for patient 1 was the use of a hearing aid. Hearing aids can be beneficial alternative treatment options due to their cost-effectiveness and non-invasiveness. Patient 2 had no otologic symptoms and there was also no evidence of hearing disability on audiogram. However, there was an evidence of demineralization change on both cochlea but not in fissula ante fenestram.

The difference in their hearing level maybe related with the difference in their $\mathrm{CT}$ findings. The greater the demineralization of the otic capsule and fissula ante fenestram, the more advanced the hearing loss. Based on their CT findings and audiograms, we recommend $\mathrm{CT}$ for both patients in addition to PTA to search for development or progression of otologic symptoms and development of demineralization of area of fissula ante fenestram in the case of patient 2.

It is important to regularly follow-up patients with otosclero- sis not only with audiograms but also with CT. This will enable the clinicians to provide timely medico-surgical management prior to recognition of the impairment of their hearing ability.

\section{Acknowledgments}

This work was supported by the National Research Foundation of Korea (NRF) grant funded by the Korea government (MSIT) (No. NRF-2020R1A2C1009789) and by National Information Society Agency (NIA) funded by the Ministry of Science, ICT.

\section{Conflicts of interest}

The authors have no financial conflicts of interest.

\section{Author Contributions}

Conceptualization: Young Joon Seo. Data curation: Young Joon Seo. Investigation: Ha Neul Lee. Methodology: Young Joon Seo. Project administration: Young Joon Seo. Resources: Young Joon Seo. Supervision: Young Joon Seo. Validation: Hyun Jong Jeon. Visualization: Ha Neul Lee. Writing — original draft: Ha Neul Lee. Writing — review \& editing: Hyun Jong Jeon and Young Joon Seo. Approval of final manuscript: all authors.

\section{ORCID iDs}

Ha Neul Lee

Hyun Jong Jeon

https://orcid.org/0000-0002-8893-1154

Young Joon Seo

https://orcid.org/0000-0002-1345-236X

https://orcid.org/0000-0002-2839-4676

\section{REFERENCES}

1) Batson L, Rizzolo D. Otosclerosis: an update on diagnosis and treatment. JAAPA 2017;30:17-22.

2) Chang SO, Kim CS, Oh SH, Park JB, Ahn SH, Hwang CH, et al. HRCT findings of otosclerosis. Korean J Otolaryngol-Head Neck Surg 2002;45:118-21.

3) Declau F, Spaendonck MV, Timmermans JP, Michaels L, Liang J, Qiu JP, et al. Prevalence of histologic otosclerosis: an unbiased temporal bone study in Caucasians. Adv Otorhinolaryngol 2007;65:6-16.

4) Ban JH, Lee SS, Kwon HJ, Lee JK. A case of familial otosclerosis. Korean J Otolaryngol-Head Neck Surg 2007;50:182-5.

5) Sillence DO, Senn A, Danks DM. Genetic heterogeneity in osteogenesis imperfecta. J Med Genet 1979;16:101-16.

6) Santos F, McCall AA, Chien W, Merchant S. Otopathology in osteogenesis imperfecta. Otol Neurotol 2012;33:1562-6.

7) Hartikka H, Kuurila K, Körkkö J, Kaitila I, Grénman R, Pynnönen $\mathrm{S}$, et al. Lack of correlation between the type of COL1A1 or COL1A2 mutation and hearing loss in osteogenesis imperfecta patients. Hum Mutat 2004;24:147-54.

8) Vincent R, Sperling NM, Oates J, Jindal M. Surgical findings and long-term hearing results in 3,050 stapedotomies for primary otosclerosis: a prospective study with the otology-neurotology database. Otol Neurotol 2006;27:S25-47.

9) Lippy WH, Berenholz LP, Schuring AG, Burkey JM. Does pregnancy affect otosclerosis? Laryngoscope 2005;115:1833-6.

10) Meyer TA, Lambert PR. Primary and revision stapedectomy in elderly patients. Curr Opin Otolaryngol Head Neck Surg 2004;12:387-92.

11) Lenarz T, Zwartenkot JW, Stieger C, Schwab B, Mylanus EA, Caversaccio M, et al. Multicenter study with a direct acoustic cochlear implant. Otol Neurotol 2013;34:1215-25.

12) Venail F, Lavieille JP, Meller R, Deveze A, Tardivet L, Magnan J. New perspectives for middle ear implants: first results in otosclerosis with mixed hearing loss. Laryngoscope 2007;117:552-5.

13) van Loon MC, Merkus P, Smit CF, Smits C, Witte BI, Hensen EF. 
Stapedotomy in cochlear implant candidates with far advanced otosclerosis: a systematic review of the literature and meta-analysis. Otol Neurotol 2014;35:1707-14.

14) Quaranta N, Bartoli R, Lopriore A, Fernandez-Vega S, Giagnotti F, Quaranta A. Cochlear implantation in otosclerosis. Otol Neurotol
2005;26:983-7.

15) Liktor B, Szekanecz Z, Batta TJ, Sziklai I, Karosi T. Perspectives of pharmacological treatment in otosclerosis. Eur Arch Otorhinolaryngol 2013;270:793-804. 